IUHET-252

UDHEP-93-03

BA-93-44

\title{
RENORMALIZATION OF THE NEUTRINO MASS OPERATOR
}

\author{
K.S. Babu ${ }^{1}$, C.N. Leung ${ }^{2}$, J. Pantaleone ${ }^{3}$ \\ ${ }^{1}$ Bartol Research Institute \\ ${ }^{2}$ Department of Physics and Astronomy \\ University of Delaware, Newark, DE 19716 \\ ${ }^{3}$ Physics Department \\ Indiana University, Bloomington, IN 47405
}

\begin{abstract}
A small neutrino Majorana mass can arise in the Standard Model as an effective dimension 5 operator. We calculate the renormalization of this operator in the minimal Standard Model and in its two-Higgs-doublet and supersymmetric extensions. Renormalization from the scale of lepton number violation (e.g., the Planck scale or a GUT scale) to the weak scale decreases the strength of this operator by an order of magnitude or more if the top quark and Higgs boson masses are large. Neutrino mixing angles also run with momentum. We show instances where small mixing at a high scale becomes large at the weak scale, and vice versa.
\end{abstract}


1. Introduction. There are several indications that physical processes do not respect the global baryon number $(B)$ and lepton number $(L)$ symmetries. For example, in the Standard Model (SM), nonperturbative effects due to instantons break the $B+L$ symmetry [1]. The observed matter-antimatter asymmetry of the universe is an explicit evidence for $B$ violation. In addition, simple arguments suggest that quantum effects of gravity may violate global symmetries [2]. It thus appears that $B$ and $L$ symmetries are not fundamental symmetries of the full theory, but are "accidents" of the low energy structure of the theory - as is the case in Grand Unified Theories (GUT). If lepton number is indeed not conserved, a neutrino Majorana mass will be generated at some level.

Let $M_{X}$ be the scale above which lepton number is broken. This could be the Planck scale (if the lepton number violation is induced only by gravity) or a GUT scale. Assuming that the only light fields below $M_{X}$ are the ones given in the SM, D then the lowest dimension operator which generates a Majorana mass for the left-handed neutrinos, $\nu_{L}$, is unique [3] :

$$
\mathcal{L}_{\nu \nu}=\frac{1}{4} \kappa_{m n} \bar{c}^{m}{ }_{L i}^{m} l_{L j}^{n} \Phi_{k} \Phi_{l} \epsilon_{i k} \epsilon_{j l}+\text { h.c. }
$$

where $l_{L}$ and $\Phi$ are the left-handed lepton and Higgs boson doublets, respectively,

$$
l_{L}^{m}=\left[\begin{array}{c}
\nu_{L}^{m} \\
e_{L}^{m}
\end{array}\right] ; \quad \Phi=\left[\begin{array}{c}
\Phi^{+} \\
\Phi^{0}
\end{array}\right] .
$$

\footnotetext{
${ }^{1}$ We do not consider here the possibility of light "exotic" particles such as triplet Higgs bosons, although later we shall consider the two-Higgs-doublet and the minimal supersymmetric extensions.
} 
$\kappa_{m n}$ is symmetric under interchange of $m$ and $n$, the generation indices; $i, j, k, l$ are $\mathrm{SU}(2)$ indices. When the Higgs scalar develops a vacuum expectation value, $\left\langle\Phi^{0}\right\rangle=v / \sqrt{2}$, the $\mathrm{SU}(2)$ gauge symmetry is spontaneously broken and the neutrino Majorana mass matrix ensues:

$$
M_{\nu}=-\frac{1}{4} \kappa v^{2}
$$

The operator in Eq. (1) has dimension 5, so $\kappa$ is of order $1 / M_{X}$. Consequently the magnitude of the neutrino masses is suppressed with respect to the charged fermion masses by the factor $v / M_{X}$.

Models for neutrino masses predict the coefficients, $\kappa_{m n}$, in terms of other parameters. For example, if $\nu_{R}$ 's exist, they should have a large Majorana mass, $M_{R}$, from lepton number breaking and hence there will be a contribution to $\kappa$ of

$$
\kappa \simeq 2 Y_{\nu}^{T} M_{R}^{-1} Y_{\nu}
$$

which is the popular see-saw mechanism [4]. Here $Y_{\nu}$ is the Yukawa coupling matrix between the $l_{L}$ 's and $\nu_{R}$ 's. This prediction is not unique. Models with more than one heavy scale (such as the GUT and Planck scales), radiative models [5, 6], or models with exotic heavy particles can give other predictions. The values of $\kappa$ depend on the short distance dynamics responsible for lepton number breaking, while the operator structure in Eq. (11) depends only on the low energy contents of the model.

Radiative corrections renormalize the neutrino mass operator, as they do all the terms in the Lagrangian. Studies of the running of the SM couplings have led to the important observation that gauge coupling constants may unify at a large scale. The "running" of the neutrino mass operator 
is also important for several reasons: 1) to correctly relate the scale of lepton number violation to other physical scales, e.g., proton decay, coupling constant unification, quantum gravity, etc." and 2) to correctly relate the mixing angles predicted by a model at high scales to the values at low scales where they are measured. However, despite the fact that the renormalization of the dimension 6 operators which contribute to proton decay have been studied to two loops [8], the running of the dimension 5 term which yields a neutrino mass has received little attention. In this note we compute the renormalization of the neutrino mass operator above the weak scale. pe first consider the standard model with one Higgs doublet. Then we shall examine its two-Higgs-doublet and supersymmetric extensions.

2. One Higgs Doublet. We choose to compute the renormalization of the neutrino mass operator, Eq. (11), in Landau gauge. The topologically distinct one-loop diagrams which give nonzero contributions are shown in Fig. 1. Beside the gauge interactions, there are the scalar quartic interactions,

$$
\mathcal{L}_{H}=-\frac{\lambda}{2}\left(\Phi^{\dagger} \Phi\right)^{2}
$$

and the Yukawa interactions,

$$
\mathcal{L}_{Y}=\bar{Q}_{L} \tilde{\Phi} Y_{u}^{\dagger} u_{R}+\bar{Q}_{L} \Phi Y_{d}^{\dagger} d_{R}+\bar{l}_{L} \Phi Y_{e}^{\dagger} e_{R}+\text { h.c. }
$$

\footnotetext{
${ }^{2}$ Solar neutrino experiments are sensitive to neutrino masses as small as $10^{-6} \mathrm{eV}$ (see, e.g., [7]). Such a small mass corresponds to lepton number breaking at a scale of order the Planck scale, $\kappa^{-1} \sim \frac{v^{2}}{4 m_{\nu}} \sim 10^{19} \mathrm{GeV}$.

${ }^{3}$ The neutrino mass does not run significantly below the weak scale because there the neutrino interactions with other light particles are suppressed by the factor $q^{2} / M_{W}^{2}$.
} 
Here $Q_{L}$ is the left-handed quark doublet,

$$
Q_{L}=\left[\begin{array}{l}
u_{L} \\
d_{L}
\end{array}\right],
$$

$\tilde{\Phi}=i \tau_{2} \Phi^{*}$ and $u_{R}, d_{R}$, and $e_{R}$ are the right-handed quark and charged lepton fields. $Y_{f}$ is the Yukawa coupling matrix of fermion species $f=u, d, e$. Generation indices have been suppressed. With the above parametrization the physical Higgs boson mass is $m_{H}^{2}=\lambda v^{2}$, where $v=246 \mathrm{GeV}$.

The evolution equation for $\kappa$ is found to be

$$
16 \pi^{2} \frac{d \kappa}{d \ln \mu}=\left[-3 g_{2}^{2}+2 \lambda+2 S\right] \kappa-\frac{1}{2}\left[\kappa\left(Y_{e}^{\dagger} Y_{e}\right)+\left(Y_{e}^{\dagger} Y_{e}\right)^{T} \kappa\right]
$$

where $\mu$ is the renormalization scale, $g_{2}$ is the $\mathrm{SU}(2)$ gauge coupling constant and

$$
S=\operatorname{Tr}\left[3 Y_{u}^{\dagger} Y_{u}+3 Y_{d}^{\dagger} Y_{d}+Y_{e}^{\dagger} Y_{e}\right]
$$

is the contribution associated with Fig. 1c.

The presence of two Higgs fields in the neutrino mass operator leads to qualitative and quantitative differences between the running of $\kappa$ and the running of the charged fermion Yukawa couplings [9]. Firstly, $\lambda$ enters Eq. (8) at leading order, so the evolution is sensitive to large Higgs boson masses. Moreover, the evolution is twice as sensitive to a large top quark mass. Both of the above terms enter Eq. (8) with the same sign, so $\kappa$ can run faster than the charged fermion Yukawa couplings do.

Solving Eq. (8), Fig. 2 plots how the magnitude of $\kappa$ evolves through the assumed desert above the weak scale. We numerically evolve all relevant coupling constants simultaneously over the entire range of $\mu$ between $M_{Z}$ 
(the $Z$ boson mass) and $M_{X}$, taken here to be near the Planck scale. (The renormalization group equations for the SM couplings can be found in, e.g., [9, 10].) The Yukawa couplings of the lightest two families have negligible effects on the running of other parameters and will be ignored henceforth. We have also ignored in Fig. 2 the small contributions from the $\tau$-lepton Yukawa coupling. In this approximation, all elements of $\kappa$ evolve identically. The parameters are chosen so that $m_{t}$ (the top quark mass) and $m_{H}$ remain small enough for perturbation theory to be valid. We take $\alpha_{s}\left(M_{Z}\right)=0.12$, $\alpha^{-1}\left(M_{Z}\right)=128$, and $\sin ^{2} \theta_{W}\left(M_{Z}\right)=0.233$. The running is significant when either $m_{t}$ or $m_{H}$ is large (but especially when $m_{t}$ is large). Large $m_{t}$ and $m_{H}$ will cause $\kappa$ to decrease by an order of magnitude or more in running from the Planck scale to the weak scale.

The renormalization of $\kappa$ can alter the predictions of neutrino mass models. For example, in $\mathrm{SO}(10)$ type theories [11, 12, 13], if the neutrino Dirac mass matrix is identified with that of the up-quarks and the Majorana mass matrix of $\nu_{R}$ is taken to be identity, the physical neutrino masses will be given by the see-saw formula

$$
m_{\nu} \simeq \frac{m_{u}^{2}}{M_{R}}\left[\frac{\kappa\left(M_{Z}\right)}{\kappa\left(M_{X}\right)}\right]\left[\frac{Y_{u}\left(M_{X}\right)}{Y_{u}\left(m_{u}\right)}\right]^{2}
$$

where $m_{u}$ denotes the physical mass of the up-type quark and $M_{R} \sim M_{X}$ is the Majorana mass of $\nu_{R}$. Assuming an intermediate scale $M_{X} \sim 10^{10} \mathrm{GeV}$ and taking into account the running of $Y_{u}$ (but not the running of $\kappa$ ), the authors of Ref. [11 obtain $m_{\nu_{e}}=0.05 \frac{m_{u}^{2}}{M_{R}}, m_{\nu_{\mu}}=0.07 \frac{m_{c}^{2}}{M_{R}}, m_{\nu_{\tau}}=0.18 \frac{m_{t}^{2}}{M_{R}}$. Including the running of $\kappa$ reduces these estimates of neutrino masses by by an additional factor of 2 to 3 for $m_{H}$ near $200 \mathrm{GeV}$. Consequently, 
the intermediate symmetry $S U(3)_{c} \times S U(2)_{L} \times S U(2)_{R} \times U(1)$ studied in Ref. [12] may become compatible with the Mikheyev-Smirnov-Wolfenstein solution [14] of the solar neutrino puzzle. In contrast, large running would spoil proposals [15] to relate the just-so solution of the solar neutrino problem (where $m_{\nu} \simeq 10^{-5} \mathrm{eV}$ ) with the Planck scale.

The running of $\kappa$ also affects model predictions of the neutrino mixing angles. In the approximation of two neutrino mixing, the mixing angle is given by

$$
\tan 2 \theta=\frac{2 \kappa_{12}}{\kappa_{22}-\kappa_{11}}
$$

in the basis where $Y_{e}$ is diagonal. Eq. (8) determines the evolution equation for the mixing angle to be

$$
16 \pi^{2} \frac{d \sin ^{2} 2 \theta}{d \ln \mu}=\sin ^{2} 2 \theta\left(1-\sin ^{2} 2 \theta\right)\left(y_{2}^{2}-y_{1}^{2}\right) \frac{\kappa_{22}+\kappa_{11}}{\kappa_{22}-\kappa_{11}},
$$

where $y_{1}$ and $y_{2}$ are the Yukawa couplings of the corresponding charged leptons. As expected, the mixing angle does not run when the mixing is maximal or zero. However, significant running can occur if the magnitude of $\kappa_{22}-\kappa_{11}$ is less than or comparable to $y_{\tau}^{2} \sim 10^{-4}$. (This is the case in some pseudo-Dirac [16] neutrino models.) As a result, large mixing at $M_{X}$ can become small mixing at the weak scale, and vice versa.

Unlike the elements of $\kappa$, large running of the mixing angle does not require a heavy top quark and/or Higgs boson. Examples are shown in Fig. 3. We have chosen $m_{t}\left(M_{Z}\right)=130 \mathrm{GeV}$ and $m_{H}\left(M_{Z}\right)=100 \mathrm{GeV}$. $y_{2}$ is taken to be the $\tau$ Yukawa coupling, so Fig. 3 is relevant for $e-\tau$ and $\mu-\tau$ mixing. Curves a) and b) show that the mixing angle can decrease drastically from its values at high energies. This can be achieved for neutrino masses 
of order $1 \mathrm{eV}$ and $\Delta m^{2}=m_{2}^{2}-m_{1}^{2}$ of order $10^{-5}$ to $10^{-6} \mathrm{eV}^{2}$. Curves c) and d) illustrate the case in which small mixing at a high scale can become large at a lower scale. This requires $\kappa_{22} \lesssim \kappa_{11}$ at the high scale, assuming $y_{2}^{2}>y_{1}^{2}$. The running of the mixing angle now depends more sensitively on the degeneracy in $\kappa$. This is because, according to Eq. (8), $\kappa_{22}$ decreases with decreasing momentum more slowly than $\kappa_{11}$. Consequently they will cross at some scale, which leads to the resonance curve shown in c).

3. Two Higgs Doublets. As is customary, we assume that the Higgs doublets transform independently under the discrete symmetry

$$
\Phi_{i} \rightarrow-\Phi_{i}
$$

for which the most general Higgs potential is

$$
\begin{aligned}
\mathcal{L}_{2 H}= & -\frac{\lambda_{1}}{2}\left(\Phi_{1}^{\dagger} \Phi_{1}\right)^{2}-\frac{\lambda_{2}}{2}\left(\Phi_{2}^{\dagger} \Phi_{2}\right)^{2}-\lambda_{3}\left(\Phi_{1}^{\dagger} \Phi_{1}\right)\left(\Phi_{2}^{\dagger} \Phi_{2}\right) \\
& -\lambda_{4}\left(\Phi_{1}^{\dagger} \Phi_{2}\right)\left(\Phi_{2}^{\dagger} \Phi_{1}\right)-\left[\frac{\lambda_{5}}{2}\left(\Phi_{1}^{\dagger} \Phi_{2}\right)^{2}+\text { h.c. }\right]
\end{aligned}
$$

The discrete symmetry is to insure that there are no flavor changing neutral Higgs couplings in the dimension 4 terms. With this symmetry, each type of charged fermions can couple to only one Higgs doublet. We denote the doublet which couples to the charged leptons as $\Phi_{1}$. However, there are now four ways to combine two Higgs fields and two neutrino fields, resulting in four operators relevant to the evolution of the neutrino mass. These operators fall into two classes according to how the product $\Phi_{i} \Phi_{j}$ transform under the discrete symmetry. Operators where $\Phi_{i} \Phi_{j}$ transform identically will be mixed by renormalization. 
The two operators in which $\Phi_{i} \Phi_{j}$ are even are

$$
\mathcal{L}_{\nu \nu}=\frac{1}{4} \kappa_{m n}^{(11)} \bar{l}_{L i}^{m} l_{L j}^{n} \Phi_{1}^{k} \Phi_{1}^{l} \epsilon_{i k} \epsilon_{j l}+\frac{1}{4} \kappa_{m n}^{(22)} \bar{l}_{L i}^{m} l_{L j}^{n} \Phi_{2}^{k} \Phi_{2}^{l} \epsilon_{i k} \epsilon_{j l}+\text { h.c. } .
$$

Calculating the class of diagrams shown in Fig. 1, we find the evolution of $\kappa^{(11)}$ and $\kappa^{(22)}$,

$$
\begin{aligned}
16 \pi^{2} \frac{d \kappa^{(11)}}{d \ln \mu} & =\left[-3 g_{2}^{2}+2 \lambda_{1}+2 S^{11}\right] \kappa^{(11)}-\frac{1}{2}\left[\kappa^{(11)}\left(Y_{e}^{\dagger} Y_{e}\right)+\left(Y_{e}^{\dagger} Y_{e}\right)^{T} \kappa^{(11)}\right]+2 \lambda_{5}^{*} \kappa^{(22)}, \\
16 \pi^{2} \frac{d \kappa^{(22)}}{d \ln \mu} & =\left[-3 g_{2}^{2}+2 \lambda_{2}+2 S^{22}\right] \kappa^{(22)}+\frac{1}{2}\left[\kappa^{(22)}\left(Y_{e}^{\dagger} Y_{e}\right)+\left(Y_{e}^{\dagger} Y_{e}\right)^{T} \kappa^{(22)}\right]+2 \lambda_{5} \kappa^{(11)} .
\end{aligned}
$$

Here $S^{r r}$ is the two-Higgs-doublet generalization of Eq. (9). Its precise form depends on which charged fermions $\Phi_{r}$ couples to. For instance, if $\Phi_{1}$ couples to the down type quarks and the charged leptons and $\Phi_{2}$ couples to the up type quarks, then

$$
S^{11}=\operatorname{Tr}\left[3 Y_{d}^{\dagger} Y_{d}+Y_{e}^{\dagger} Y_{e}\right], \quad S^{22}=\operatorname{Tr}\left[3 Y_{u}^{\dagger} Y_{u}\right]
$$

The two operators in which $\Phi_{i} \Phi_{j}$ are odd are

$$
\mathcal{L}_{\nu \nu}=\frac{1}{2} \kappa_{m n}^{(12)} \bar{l}_{L i}^{m} l_{L j}^{n} \Phi_{1}^{k} \Phi_{2}^{l}\left(\epsilon_{i k} \epsilon_{j l}-\frac{1}{2} \epsilon_{i j} \epsilon_{k l}\right)+\frac{1}{2} \xi_{m n}^{(12)} \bar{l}^{c}{ }_{L i}^{m} l_{L j}^{n} \Phi_{1}^{k} \Phi_{2}^{l} \epsilon_{i j} \epsilon_{k l}+\text { h.c. }
$$

Here $\kappa_{m n}^{(12)}$ (and all previous $\kappa$ 's) are symmetric under interchange of the generation indices $m$ and $n$, while $\xi_{m n}^{(12)}$ is antisymmetric. Again calculating the class of diagrams shown in Fig. 1, we find the evolution equations

$$
\begin{aligned}
16 \pi^{2} \frac{d \kappa^{(12)}}{d \ln \mu}= & {\left[-3 g_{2}^{2}+2 \lambda_{3}+2 \lambda_{4}+S\right] \kappa^{(12)}-\frac{1}{2}\left[\kappa^{(12)}\left(Y_{e}^{\dagger} Y_{e}\right)+\left(Y_{e}^{\dagger} Y_{e}\right)^{T} \kappa^{(12)}\right] } \\
& +2\left[\xi^{(12)}\left(Y_{e}^{\dagger} Y_{e}\right)-\left(Y_{e}^{\dagger} Y_{e}\right)^{T} \xi^{(12)}\right], \\
16 \pi^{2} \frac{d \xi^{(12)}}{d \ln \mu}= & {\left[-9 g_{2}^{2}+2 \lambda_{3}-2 \lambda_{4}+S\right] \xi^{(12)}+\frac{3}{2}\left[\xi^{(12)}\left(Y_{e}^{\dagger} Y_{e}\right)+\left(Y_{e}^{\dagger} Y_{e}\right)^{T} \xi^{(12)}\right] } \\
& +\frac{3}{2}\left[\kappa^{(12)}\left(Y_{e}^{\dagger} Y_{e}\right)-\left(Y_{e}^{\dagger} Y_{e}\right)^{T} \kappa^{(12)}\right] .
\end{aligned}
$$


Only the $\kappa^{(12)}$ operator contains a neutrino mass term, however the above equations show that the two operators are mixed by renormalization and so $\xi^{(12)}$ must also be simultaneously evolved.

Different neutrino mass models yield different subsets of these four operators below the lepton number breaking scale, $M_{X}$. For example, the see-saw mechanism in some $\mathrm{SO}(10)$ models may produce $\kappa^{(22)}$ and $\kappa^{(12)}$ while the Zee model [6] produces $\kappa^{(12)}$ and $\xi^{(12)}$. In fact, one can easily construct a see-saw type model which leads to any desired subset of the four operators by carefully choosing how the different $\nu_{R}$ 's transform under the discrete symmetries. After the operators are produced, they mix under evolution according to Eqs. (16) and (19) given above.

To illustrate the evolution of the neutrino mixing angles in two-Higgsdoublet models, we assume that $\Phi_{1}$ couples to the charged leptons and the down type quarks, while $\Phi_{2}$ couples to the up type quarks. We take the neutrino mass matrix to be

$$
M_{\nu}=-\frac{1}{4} v^{2}\left[\kappa^{(11)} \cos ^{2} \beta+\kappa^{(22)} \sin ^{2} \beta\right]
$$

as might occur in a see-saw model. Here $v_{i} / \sqrt{2}$ is the vacuum expectation value of $\Phi_{i}, \tan \beta=v_{2} / v_{1}$, and $v^{2}=v_{1}^{2}+v_{2}^{2}$. The parameter $\tan \beta$ runs according to

$$
16 \pi^{2} \frac{d \tan \beta}{d \ln \mu}=\tan \beta\left(S^{11}-S^{22}\right) .
$$

In general, the evolution of the neutrino mixing angles depends on the top quark and Higgs boson masses, so large changes during running are possible.

\footnotetext{
${ }^{4}$ Both even and odd operators can be produced simultaneously if the discrete symmetry is broken at $M_{X}$.
} 
To illustrate a particularly interesting possibility, we have plotted in Fig. 4 the mixing angle for a two generation system as a function of momentum. The evolution equations for the Yukawa and the quartic scalar couplings can be found, e.g., in [10]. We have chosen the parameters at the weak scale to be $y_{t}=1.35, \lambda_{1}=0.16, \lambda_{2}=1.13, \lambda_{3}=-0.0081, \lambda_{4}=-0.061, \lambda_{5}=-0.011$, and $\tan \beta=1$. This corresponds to $m_{t}=168 \mathrm{GeV}$ and a Higgs boson spectrum of $m_{H^{ \pm}}=47 \mathrm{GeV}, m_{P}=26 \mathrm{GeV}, m_{h}=67 \mathrm{GeV}, m_{H}=186 \mathrm{GeV}$, where $H^{ \pm}, P, h$ and $H$ stand for the charged, pseudoscalar, and the two scalar Higgs bosons. Such a spectrum is phenomenologically acceptable and also guarantees that the Higgs potential remains bounded throughout the entire momentum range up to $M_{X}$, taken here to be $10^{14} \mathrm{GeV}$. Our choice of parameters is such that $y_{t}$ and $\lambda_{2}$ are near their infrared fixed point values while others are not. We then choose the $\kappa$ 's to have, in accordance with naive expectations, a large hierarchy at $M_{X}: \kappa_{11}^{(11)}=0.05, \kappa_{12}^{(22)}=0.05, \kappa_{22}^{(22)}=1.0$ (in suitable units) and all other elements to be zero. For this parameter choice, there occurs at the weak scale a degeneracy between the diagonal elements of the neutrino mass matrix. At this "resonance" the mixing is maximal. The dashed line in Fig. 4 is the evolution for all the same parameters except $\kappa_{11}^{(11)}=0.06$. Now the resonance occurs at a higher momentum. These curves illustrate that small mixing at the high scale can become large and even maximal mixing at the weak scale. With suitable choice of the parameters, the opposite can also happen, i.e., large mixing at the high scale can become small at the weak scale.

The evolution of the elements of $\kappa^{(11)}$ and $\kappa^{(22)}$ is displayed in Fig. 5. (All elements of $\kappa^{(11)}$ evolve identically if the small $\tau$ Yukawa coupling is ignored, 
similarly for $\kappa^{(22)}$.) Notice that a large variation in $\kappa^{(22)}$ (by a factor of 20 ) is now possible even for moderate values of $m_{t}$. It is clear from Fig. 5 that, unlike in the standard model, "resonant mixing" can occur in the two-Higgsdoublet models even for nondegenerate neutrinos. The reason is that the variation of $\kappa^{(22)}$ is more prominent than $\kappa^{(11)}$, since $\lambda_{2}$ and $y_{t}$ are large while $\lambda_{1}$ is not. As a result, although $\kappa_{22}^{(22)} \gg \kappa_{11}^{(11)}$ at $M_{X}$, their values can become closer at lower momenta, as can be seen from Fig. 5. The momentum scale at which mixing angle resonance occurs does not correspond to the momentum at which the two $\kappa$ 's cross, since the running of $\tan \beta$ also affects the evolution of the mixing angle.

4. Minimal Supersymmetric Standard Model (MSSM). In the MSSM, when $R$-Parity is assumed to be an exact symmetry, lepton number violation can arise only through the dimension 5 superpotential term

$$
W=\frac{1}{4} \kappa_{m n}^{(s)} L_{i}^{m} L_{j}^{n} H_{2 k} H_{2 l} \epsilon_{i k} \epsilon_{j l}
$$

Here $H_{2}$ is the $Y=1 / 2$ Higgs superfield and $L$ the leptonic doublet superfield. Unlike in the non-SUSY two-Higgs-doublet model, the operator in Eq. (22) does not mix with any other operators. The evolution equation for $\kappa^{(s)}$ is found to be

$$
16 \pi^{2} \frac{d \kappa^{(s)}}{d \ln \mu}=\kappa^{(s)}\left[-2 g_{1}^{2}-6 g_{2}^{2}+6 \operatorname{Tr}\left(Y_{u}^{\dagger} Y_{u}\right)\right]+\kappa^{(s)} Y_{e}^{\dagger} Y_{e}+\left(Y_{e}^{\dagger} Y_{e}\right)^{T} \kappa^{(s)}
$$

For a two-family system, the mixing angle evolution is obtained by multiplying the right-hand side of Eq. (11) by $(-2)$. In Fig. 6 we plot the

\footnotetext{
${ }^{5}$ There are five other dimension 5 operators in the MSSM: $\left(H_{1} H_{2}\right)^{2}, Q u^{c} L e^{c}$, $Q Q u^{c} d^{c}, Q Q Q L$ and $u^{c} u^{c} d^{c} e^{c}$. All these carry zero $B-L$ charge and do not mix with the operator of Eq. (22) which has $B-L=-2$.
} 
running of $\sin ^{2} 2 \theta$ as a function of $\mu$. The parameter choice corresponds to $y_{t}=y_{b}=y_{\tau}=3$ at $M_{X}=10^{16} \mathrm{GeV}$, so that $\tan \beta=m_{t} / m_{b}$. The corresponding top-quark mass is $m_{t}=184 \mathrm{GeV}$ at the weak scale. We choose for the solid curve $\kappa_{22}^{(s)}=1, \kappa_{12}^{(s)}=0.1$ and $\kappa_{11}^{(s)}=0$ at $M_{X}$, which exhibits a large hierarchy. $\sin ^{2} 2 \theta$ is seen to increase by about a factor of 2 in running from $M_{X}$ to $M_{Z}$. The dotted curve corresponds to $\kappa_{22}^{(s)}=1, \kappa_{12}^{(s)}=0.035, \kappa_{11}^{(s)}=0.65$ at $M_{X}$. Since $y_{\tau}$ in the MSSM is larger than in the SM, the running of the mixing angle in MSSM is enhanced. Resonant mixing can occur even without degenerate neutrinos.

5. Conclusions. We have calculated the renormalization of the neutrino mass operator in the standard model with one and two Higgs doublets, and also in the minimal supersymmetric standard model. The renormalization group equations are given in Eqs. (8), (16), (19) and (23). These renormalization effects should be included in any neutrino mass model. In addition, we have solved these equations for certain parameter choices to demonstrate some interesting features. If the top quark mass and/or Higgs boson mass are large, it is generally true that the neutrino mass operators will have sizeable evolution. Their strength can decrease by an order of magnitude in running from the Planck scale to the weak scale. Furthermore, running over this momentum range can drastically change the neutrino mixing angles; possibly from small values at high energies to large values at observable scales, and vice versa. Large evolution of the neutrino mixing angle requires a near degeneracy of the neutrino masses in the standard model, but a degeneracy is not required in models with two Higgs doublets.

Note added. While this work was being completed we received reference 
[17] which discusses the same issues. Our results for the evolution equations in the supersymmetric model are in agreement. However, there are some differences for the standard model and the two-Higgs-doublet models, presumably due to the neglect of our Fig. If in Ref. [17]. Our analysis is more general and reveals the interesting possibility of resonant running of the neutrino mixing angles.

Acknowledgements. JP thanks the theory group at the University of Delaware for its hospitality. This work is supported in part by the U.S. Department of Energy under grants No. DE-FG02-84ER40163, DE-FG0291ER40661 and DE-FG02-91-ER406267. 


\section{References}

[1] G. 'tHooft, Phys. Rev. Lett. 37 (1976) 8. For a recent review, see P. Arnold, TASI 90 (1990) 719.

[2] J. D. Beckenstein, Phys. Rev. D 5 (1972) 1239; S. W. Hawking, Phys. Rev. D 14 (1976) 2460; Ya. B. Zeldovich, Sov. Phys. JETP 45 (1977) 9. For a recent discussion see J. Preskill, Proceedings of the Int. Symp. on Gravity, the Woodlands, Texas (1992).

[3] S. Weinberg, Phys. Rev. Lett. 43 (1979) 1566.

[4] M. Gell-Mann, P. Ramond, and R. Slansky, "Supergravity", Eds. P. Van Nieuwenhuizen and D.Z. Freedman (North Holland, Amsterdam) (1979) p. 315; T. Yanagida, Proceedings of the Workshop on Unified Theory and Baryon Number of the Universe, Tsukuba, Ibaraki, Japan unpublished (1979); R.N. Mohapatra and G. Senjanovic, Phys. Rev. Lett. 44 (1980) 912.

[5] E. Witten, Phys. Lett. B 91 (1980) 81.

[6] A. Zee, Phys. Lett B 93 (1980) 389; Nucl. Phys. B 264 (1986) 99.

[7] T.K. Kuo and J. Pantaleone, Rev. Mod. Phys. 61 (1988) 937.

[8] L.F. Abbot and M.B. Wise, Phys. Rev. D 22 (1980) 2208; J. Ellis, M.K. Gaillard and D.V. Nanopoulos, Nucl. Phys. B135 (1978) 66; F. Wilczek and A. Zee, Phys. Rev. Lett. 43 (1979) 1571; M. Daniel and J.A. Penarrocha, Nucl. Phys. B236 (1984) 467. 
[9] T.P. Cheng, E. Eichten and L.F. Li, Phys. Rev. D 9 (1974) 2259.

[10] C. T. Hill, C. N. Leung and S. Rao, Nucl. Phys. B262 (1985) 517.

[11] S. Bludman, D. Kennedy and P. Langacker, Phys. Rev. D 45 (1992) 1810.

[12] R.N. Mohapatra and M.K. Parida, Phys. Rev. D 47 (1992) 264.

[13] K.S. Babu and R.N. Mohapatra, Phys. Rev. Lett. 70 (1993) 2845; S. Dimopoulos, L. Hall and S. Raby, Phys. Rev. D 47 (1993) 3697.

[14] L. Wolfenstein, Phys. Rev. D 17 (1978) 2369; S. P. Mikheyev and A. Yu. Smirnov, Yad. Phys. 42 (1985) 1441 (Sov. J. Nucl. Phys. 42 (1985) 913).

[15] E. Akhmedov, Z. Berezhiani and B. Senjanovic, Phys. Rev. Lett. 69 (1992) 3013; R. Barbieri, J. Ellis and M.K. Gaillard, Phys. Lett. B90 (1980) 249.

[16] L. Wolfenstein, Nucl. Phys. B186 (1981) 147.

[17] P.H. Chankowski and Z. Pluciennik, University of Zurich preprint ZUTH 20/93. 


\section{FIGURE CAPTION}

Fig. 1 One-loop Feynman diagrams contributing to the renormalization of $\kappa$ in Landau gauge.

Fig. 2 Running of $\kappa$ below $M_{X}=10^{19} \mathrm{GeV}$ for the standard model with one Higgs doublet. The top quark and Higgs boson masses are chosen to be, respectively, in GeV: a) 226 and 243.2, b) 220 and 233.8, c) 180 and 183, d) 130 and 160, e) 130 and 100.

Fig. 3 Illustration of the running of the neutrino mixing angle in the standard model. Parameter choices are: a) $\kappa_{11}\left(M_{X}\right)=1.0$ (in suitable units), $\kappa_{22}\left(M_{X}\right)-\kappa_{11}\left(M_{X}\right)=10^{-6}$, and $M_{X}=7.88 \times 10^{18} \mathrm{GeV}$; b) same as curve a) except $\kappa_{22}\left(M_{X}\right)-\kappa_{11}\left(M_{X}\right)=10^{-5} ;$ c) $\kappa_{11}\left(M_{X}\right)=1.0001$ and $\kappa_{22}\left(M_{X}\right)=1.0$; d) $\kappa_{11}\left(M_{X}\right)=1.0002$ and $\kappa_{22}\left(M_{X}\right)=1.0$

Fig. 4 Examples of the running of the neutrino mixing angle in two-Higgsdoublet models. Parameter choices are given in the text.

Fig. 5 The running of $\kappa^{(11)}$ and $\kappa^{(22)}$ corresponding to the dotted curve in Fig. 4.

Fig. 6 Examples of the evolution of the neutrino mixing angle in the MSSM. See text for the choice of parameters. 